University of New Hampshire

University of New Hampshire Scholars' Repository

Space Science Center

Institute for the Study of Earth, Oceans, and

Space (EOS)

10-19-1999

\title{
Development and testing of a fiber/multianode photomultiplier system for use on FiberGLAST
}

\author{
Keith R. Rielage \\ Washington University in St Louis \\ Katsushi Arisaka \\ University of California - Los Angeles \\ Muzaffer Atac \\ University of California - Los Angeles \\ W Robert Binns \\ Washington University in St Louis \\ J Buckley

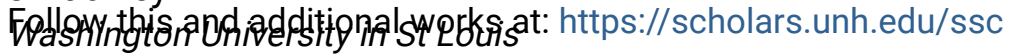 \\ Part of the Astrophysics and Astronomy Commons
}

See next page for additional authors

\section{Recommended Citation}

Keith R. Rielage ; Katsushi Arisaka ; Muzaffer Atac ; W. Robert Binns ; James H. Buckley ; Michael L. Cherry ; Mark J. Christl ; David B. Cline ; Paul F. Dowkontt ; John W. Epstein ; Gerald J. Fishman ; T. Gregory Guzik ; Paul L. Hink ; Martin H. Israel ; S. C. Kappadath ; Gerald Karr ; Richard M. Kippen ; Daniel Leopold ; Mark L. McConnell ; John R. Macri ; Robert S. Mallozzi ; William S. Paciesas ; Thomas A. Parnell ; Geoffrey N. Pendleton ; Surasak Phengchamnan ; Yuriy Pischalnikov ; Georgia A. Richardson ; James M. Ryan ; John G. Stacy ; Tumay O. Tumer ; Gerald J. Visser ; Donald B. Wallace and Robert B. Wilson "Development and testing of a fiber/multianode photomultiplier system for use on FiberGLAST", Proc. SPIE 3768, Hard X-Ray, Gamma-Ray, and Neutron Detector Physics, 156 (October 19, 1999); doi:10.1117/12.366579; http://dx.doi.org/10.1117/12.366579

This Conference Proceeding is brought to you for free and open access by the Institute for the Study of Earth, Oceans, and Space (EOS) at University of New Hampshire Scholars' Repository. It has been accepted for inclusion in Space Science Center by an authorized administrator of University of New Hampshire Scholars' Repository. For more information, please contact Scholarly.Communication@unh.edu. 


\section{Authors}

Keith R. Rielage, Katsushi Arisaka, Muzaffer Atac, W Robert Binns, J Buckley, M L. Cherry, Mark J. Christl, David B. Cline, Paul F. Dowkontt, John W. Epstein, Gerald J. Fishman, T G. Guzik, P L. Hink, Martin H. Israel, S C. Kappadath, Gerald Karr, R M. Kippen, Daniel Leopold, Mark L. McConnell, John R. Macri, Robert S. Mallozzi, W Paciesas, Thomas A. Parnell, Geoffrey N. Pendleton, Surasak Phengchamnan, Yuriy Pischalnikov, Georgia A. Richardson, James M. Ryan, J G. Stacy, O T. Tumer, Gerald J. Vissor, Donald B. Wallace, and Robert B. Wilson 


\title{
Development and testing of a fiber/multianode photomultiplier system for use on FiberGLAST
}

\author{
Keith Rielage $^{\mathrm{a}}$, Katsushi Arisaka ${ }^{\mathrm{b}}$, Muzaffer Atac ${ }^{\mathrm{b}}$, W. Robert Binns ${ }^{\mathrm{a}}$, James H. Buckley ${ }^{\mathrm{a}}$, \\ Michael L. Cherry ${ }^{c}$, Mark J. Christl ${ }^{\mathrm{d}}$, David Cline ${ }^{\mathrm{b}}$, Paul Dowkontt ${ }^{\mathrm{a}}$, John W. Epstein ${ }^{\mathrm{a}}$, \\ Gerald J. Fishman ${ }^{d}$, T.Gregory Guzik ${ }^{c}$, Paul L. Hink ${ }^{\mathrm{a}}$, Martin H. Israel ${ }^{\mathrm{a}}$, S.Cheenu Kappadath ${ }^{\mathrm{c}}$, \\ Gerald Karr $^{\mathrm{e}}$, R.Marc Kippen ${ }^{\mathrm{e}}$, Daniel Leopold ${ }^{\mathrm{a}}$, Mark L. McConnell ${ }^{\mathrm{f}}$, John Macri ${ }^{\mathrm{f}}$, Robert S. Mallozzi ${ }^{\mathrm{e}}$, \\ William S. Paciesas ${ }^{\mathrm{e}}$, Thomas A. Parnell ${ }^{\mathrm{e}}$, Geoffrey N. Pendleton ${ }^{\mathrm{e}}$, Surasak Phengchamnan ${ }^{\mathrm{e}}$, \\ Yuriy Pischalnikov ${ }^{b}$, Georgia A. Richardson ${ }^{\mathrm{e}}$, James M. Ryan ${ }^{\mathrm{f}}$, John G. Stacy $^{\mathrm{c}, \mathrm{g}}$, Tumay O. Tumer ${ }^{\mathrm{h}}$, \\ Gerard J. Visser, Donald B. Wallace ${ }^{\mathrm{e}}$, Robert B. Wilson ${ }^{\mathrm{d}}$ \\ a'Dept of Physics and McDonnell Center for Space Sciences, Washington University, \\ St. Louis, MO 63130 \\ ${ }^{b}$ Dept of Physics and Astronomy, University of California, Los Angeles, CA 90095 \\ ${ }^{c}$ Dept of Physics, Louisiana State University, Baton Rouge, LA 70803 \\ ${ }^{\mathrm{d}}$ NASA/Marshall Space Flight Center, Huntsville, AL 35812 \\ ${ }^{\mathrm{e}}$ University of Alabama in Huntsville, Huntsville, AL 35899 \\ ${ }^{\mathrm{f}}$ Space Science Center, University of New Hampshire, Durham, NH 03824 \\ ${ }^{\mathrm{g} D e p t}$ of Physics, Southern University, Baton Rouge, LA 70813 \\ ${ }^{\mathrm{h}}$ Institute of Geophysics and Planetary Physics, University of California, Riverside, CA 92521 \\ ${ }^{\mathrm{i}}$ NOVA Research and Development, Riverside, CA
}

\begin{abstract}
A scintillating fiber detector (FiberGLAST) is currently being studied for the NASA Gamma-Ray Large Area Space Telescope (GLAST) mission. This detector utilizes modules composed of a thin converter sheet followed by an $\mathrm{x}, \mathrm{y}$ plane of scintillating fibers to examine the shower of particles created by high energy gamma-rays interacting in the converter material. The detector is composed of a tracker with 90 such modular planes and a calorimeter with 36 planes. The two major components of this detector are the scintillating fibers and their associated photodetectors. Here we present current status of development and test results of both of these. The Hamamatsu R5900-00-M64 multianode (64 anodes) photomultiplier tube (MAPMT) is the baseline readout device. A characterization of this device has been performed including noise, cross-talk, gain variation, vibration, and thermal/vacuum tests. A prototype fiber/MAPMT system has been tested at the Center for Advanced Microstructures and Devices (CAMD) at Louisiana State University with a photon beam and preliminary results are presented.
\end{abstract}

Keywords: GLAST, scintillating fibers, multianode photomultiplier tube, MAPMT, radiation damage, gamma-ray astronomy

\section{OVERVIEW OF FIBERGLAST INSTRUMENT CONCEPT}

The FiberGLAST instrument is composed of three main detectors: the tracker, the calorimeter, and the anti-coincidence shield (see figure 1). Ninety detector modules spaced $2 \mathrm{~cm}$ vertically comprise the tracker. Each module includes a thin sheet of tantalum foil that stimulates pair production of high-energy photons. Directly beneath this sheet are two orthogonal layers of scintillating fibers with an active area of $1.7 \mathrm{~m}^{2}$ used to detect the passage of ionizing particles. Plastic honeycomb or foam material is used as a substrate and for the mechanical support of the module. Each fiber is read out by an anode of a multianode photomultiplier tube (MAPMT).

Contact Author: K. Rielage, email: keith@cosray2.wustl.edu 
Incident gamma rays penetrating into the instrument interact with the tantalum sheet by pair production. These electron-positron pairs are then detected by the scintillating fibers which provide the $x-y$ position in the detector. As the pair proceeds through the tracker's ninety layers, the electromagnetic shower is imaged and can be reconstructed in three dimensions. The tracker has nearly two radiation lengths of interaction material $\left(1 / 50^{\text {ith }}\right.$ r.l. thickness for each module).

The calorimeter is composed of thirty-six detector modules with thicker tantalum sheets $\left(1 / 7^{\text {th }}\right.$ r.l.), spaced $0.5 \mathrm{~cm}$ apart in the vertical direction. Two fibers will be read out by each anode of a MAPMT and the signal will be pulse height analyzed to provide energy deposition information. The calorimeter contains $\sim 5$ radiation lengths of interaction material.

The anti-coincidence system utilizes plastic scintillator panels that completely cover all four sides. top and bottom of the detector stack. Each panel consists of two layers of orthogonal plastic scintillator elements read out by waveshifter bars coupled to photomultiplier tubes. The system of segmented panels will provide the onboard computer with topological trigger information to distinguish and veto incident charged particle events from good events (Pendleton 1999).

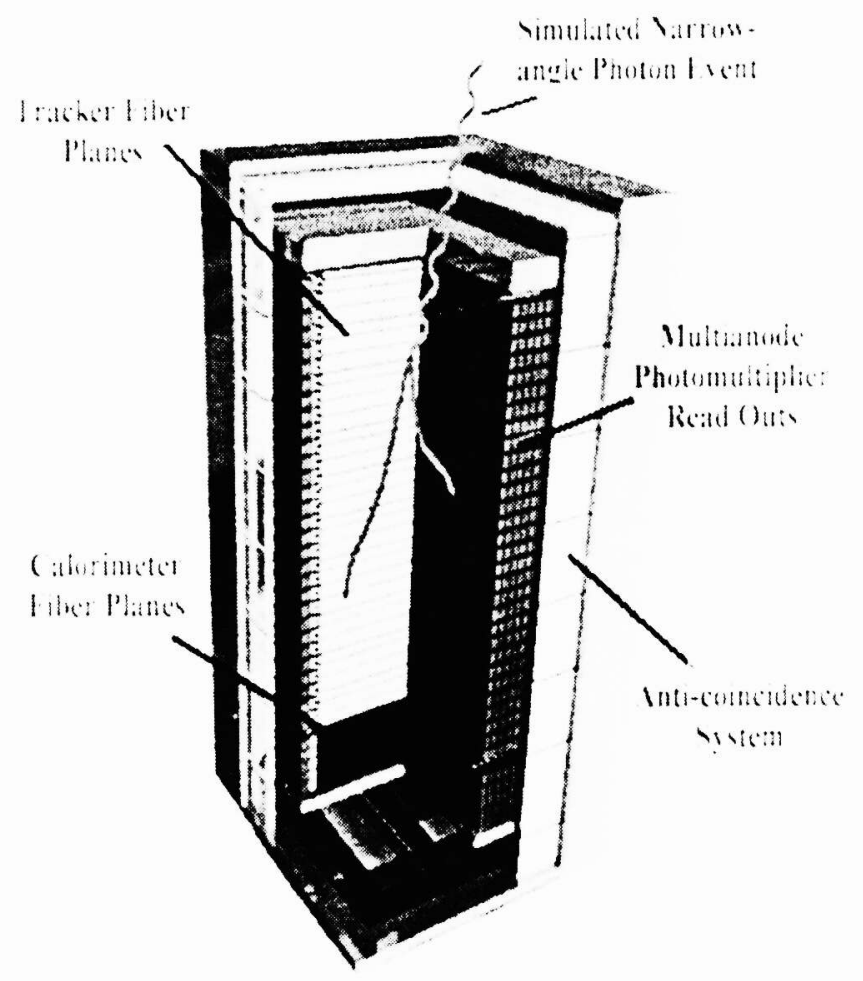

Figure 1 - FiberGLAST Instrument Overview

\section{MULTIANODE PHOTOMULTIPLIER TUBE}

\subsection{MAPMT Description}

The Hamamatsu R5900-M64 photomultiplier tube (figure 2) has 64 separate anodes arranged in an $8 \times 8$ array. Each anode is a $2 \mathrm{~mm} \times 2 \mathrm{~mm}$ square on a $2.3 \mathrm{~mm}$ pitch. The tube has an $0.8 \mathrm{~mm}$ thick borosilicate glass window over a bialkali photocathode. The tube uses 12 stage metal channel dynodes with two dynode chains for each anode. The tube has a typical gain of $10^{6}$ when operated at -900 volts. The tube can be operated at negative high voltage for DC-coupled anode output. The metal tube envelope has external dimensions of $25.7 \mathrm{~mm} \times 25.7 \mathrm{~mm} \times 20.1 \mathrm{~mm}$ and is held at cathode potential. The MAPMT has flange at the rear of the tube which is $2.8 \mathrm{~mm} \times 2.8 \mathrm{~mm}$. The quantum efficiency at $420 \mathrm{~nm}$ is $\sim 25 \%$.

\subsection{Characteristics of the R5900-M64}

Several studies have been conducted on the Hamamatsu R5900M64 (64 anodes) and R5900-M16 ( 16 anodes) MAPMTs. Enkelmann, et al. (1998) examined the tube's use for reading out scintillating fibers. The study compared the gain, uniformity, cross-talk and behavior in a magnetic field for both the M16 and the M64 versions. Since this study, Hamamatsu has continued to make improvements to the tube particularly in quantum efficiency and collection efficiency of the dynode chain. We have performed a series of tests to characterize the R5900-M64 including gain, gain uniformity, dark count, thermal effects, electrical cross-talk, single photoelectron response, and thermalvacuum tests.

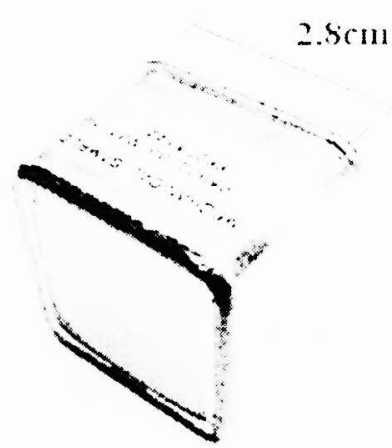

Figure 2 - Multianode photmultiplier tube 


\subsubsection{Uniformity}

The anode to anode gain variation of the M64 was tested using a blue LED producing a low light level at a distance of $25 \mathrm{~cm}$ from the tube which illuminated the whole photocathode to provide single photoelectron events. All 64 anodes were read out in parallel. The relative gain of each anode was determined from the single photoelectron peak in the pulse height distribution. A custom pulse height analyzer was used. The gain variation between anodes for the thirty tubes measured was approximately a factor of three (Figure 3 shows variation for 16 tubes including several tubes not meeting the 3:1 specification). The gain of the MAPMT was measured as a function of voltage. Using an LED which provided single photoelectron we measured the gain of the M64 for voltages between 850 and 1000 volts (see figure 4). Gains of $6.22 \times 10^{5}$ and $3.48 \times 10^{6}$ were found for cathode voltages of $-850 \mathrm{~V}$ and $-1000 \mathrm{~V}$ respectively.

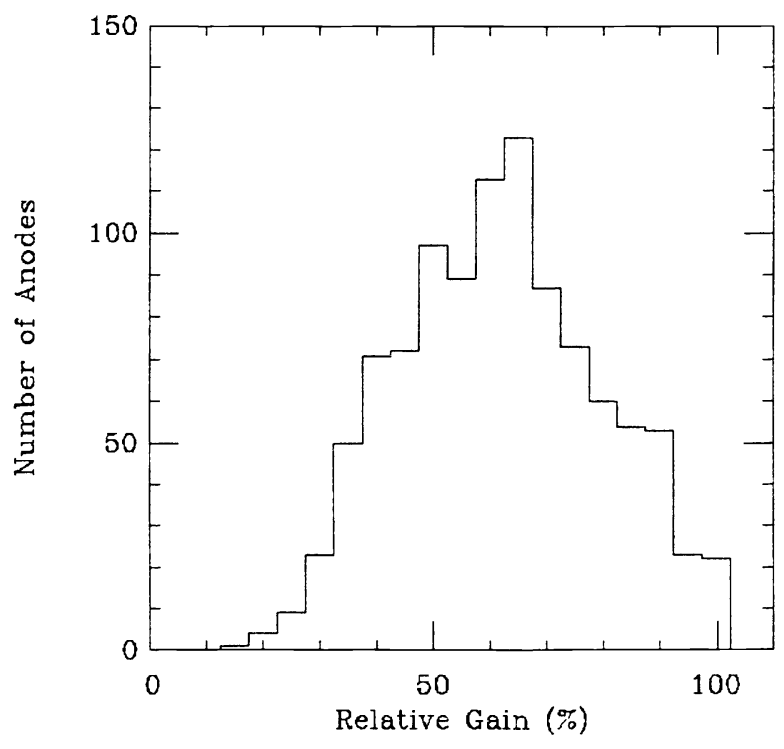

Figure 3 - Relative gain of anodes for 16 tubes (1024 anodes total)

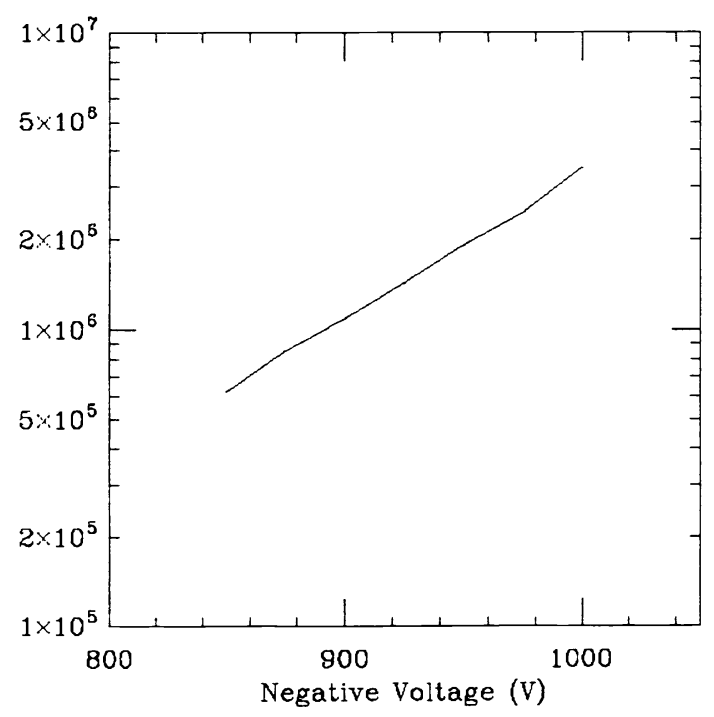

Figure 4- Gain of typical tube for single photoelectron signal

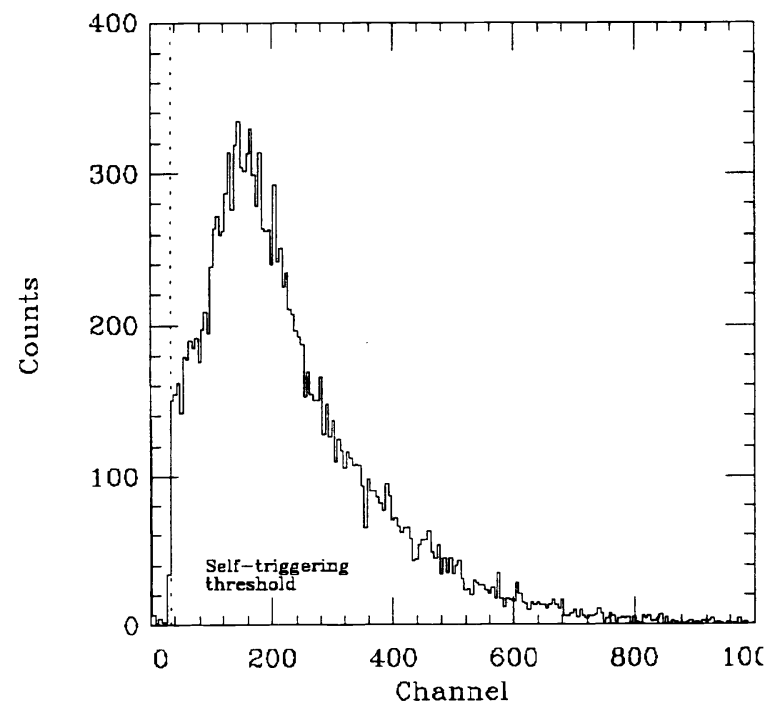

Figure 5 - Typical single photoelectron spectra - self-triggered 


\subsubsection{Single Photoelectron Response}

Figure 5 shows the pulse height distribution of single photoelectron events for a single typical self-triggered anode. The discriminator is set at a level of 0.2 photoelectrons and this clearly shows that the MAPMT has a high single photoelectron detection efficiency. Single photoelectron response does vary to some extent as a function of anode position and will be studied in more detail in the future.

\subsubsection{Dark count}

The dark count is one of the most important properties of a sensor in a system such as FiberGLAST with a large number of detector elements since, for a given coincidence resolving time, it contributes to the "occupancy" (the number of anodes that have a signal in a given event) of the system. The dark count was measured as a function of temperature. Using a settable discriminator, the summed dark count of eight anodes was measured for three different sets of anodes after being in the dark for over one hour. The average dark count per anode per second was then computed and plotted versus temperature (figure 6). At $25^{\circ} \mathrm{C}$ the average dark count is $<1$ counts/anode/second for a discriminator setting corresponding to 0.2 pe's (this is the same level used for the self-triggering threshold used in figure 5). Thus we see that for a detector such as FiberGLAST with $4 \times 10^{6}$ anodes and a $1 \mathrm{~ms}$ coincidence resolving time, the occupancy due to dark counts is $\sim 4$ at room temperature. Figure 7 shows a typical anode pulse height spectrum taken for a five minutes duration of an LED flashing at a single photoelectron level and the corresponding five minute dark count spectra at room temperature. Only 275 dark counts were measured in the five minute duration. This clearly shows that event readout time is not artificially supressing the dark count rate.

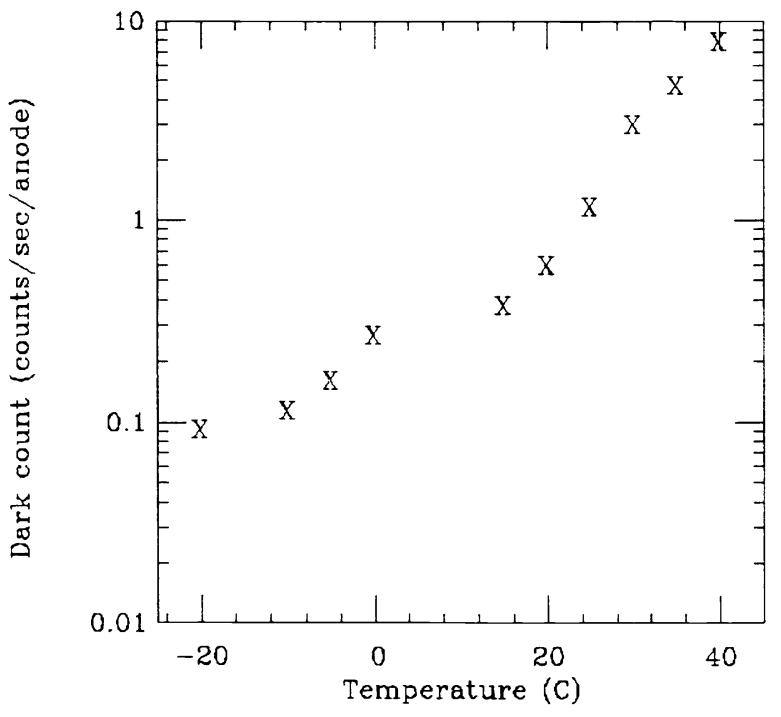

Figure 6 - Average dark count for an anode

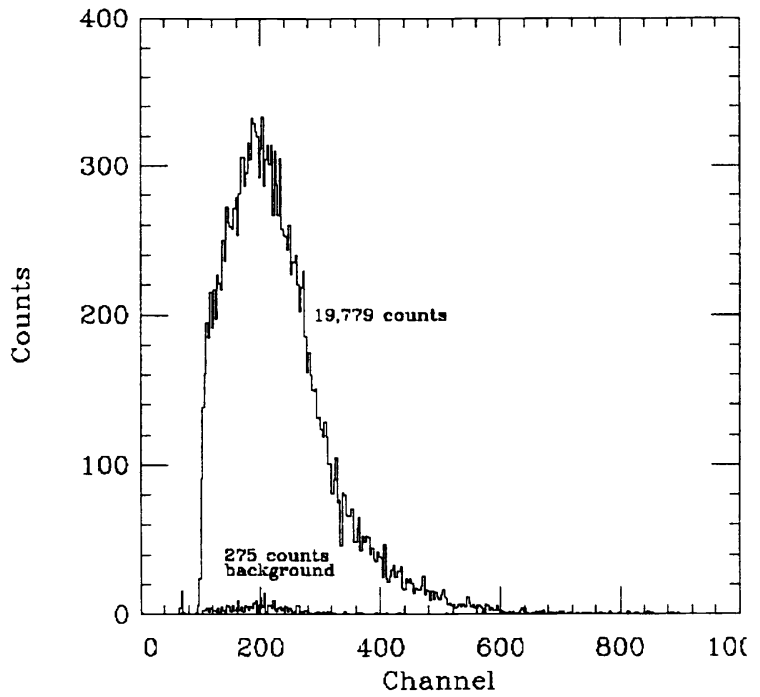

Figure 7 - Dark count and signal in 5 minutes for a) single p.e. events and $b$ ) dark count events

LED

\subsubsection{Anode Cross-talk}

Electrical cross-talk was measured by using a blue LED at a low light level to illuminate the whole photocathode with mostly single photoelectron events. The read out electronics were set up to allow any of the 64 anodes to trigger a read of the pulse height analyzer boards. A crossplot of two adjacent anode signals is shown in figure 8 . The signal on anode 25 is plotted against the signal on anode 26 for each event. The points centered at 65,65 are pedestal events where neither anode had a signal and the read was triggered by another anode. The horizontal line at $y=\sim 65$ is formed by events where anode 25 has a signal and anode 26 is at pedestal plus whatever cross-talk might result from the signal on anode 25 . Likewise, the vertical line at $x=\sim 65$ is formed by events where anode 26 has a non-zero signal and anode 25 is at pedestal plus whatever 
cross-talk might result from the signal on anode 26. The points on this vertical line split into two distinct lines as the signal on anode 26 increases. This is a result of cross-talk from signal appearing on anode 25 . This "cross-talk" signal appears roughly half the time and is $\sim 2 \%$ of the total signal on anode 26 . This cross-talk is only seen on the anode immediately to the left of the anode with signal. There is no such feature observed in anodes above, below, or to the right of the anode with signal. As is stated above, each anode is fed by two dynode chains. We believe that the observed split is electrical cross-talk due to the cupped configuration of the final metal channel dynode stage which allows the left chain to deposit $\leq 2 \%$ of its electrons onto the adjacent anode. The effect is seen for only half of the events since the right chain's $2 \%$ cross-talk is still deposited on the correct anode. Exact diagrams of the metal channel dynode stages are not available due to their proprietary nature.

Measurements of optical cross-talk within the MAPMT have also been made using a $0.75 \mathrm{~mm}$ square blue-emitting scintillating fiber centered on an anode. Our measurements give an upper limit of $2 \%$ of cross-talk on each of the four adjacent anodes. This is consistent with the measurement of $0.9 \%$ made by Enckelmann et al. (1998) using a $1.5 \mathrm{~mm}$ round fiber. This optical cross-talk is due to the thickness of the borosilicate glass window $(0.8 \mathrm{~mm})$ and the distance between it and the photocathode. These measurements are also consistent with those made by the manufacturer. We are currently making additional measurements to characterize optical cross-talk within the tube more precisely.

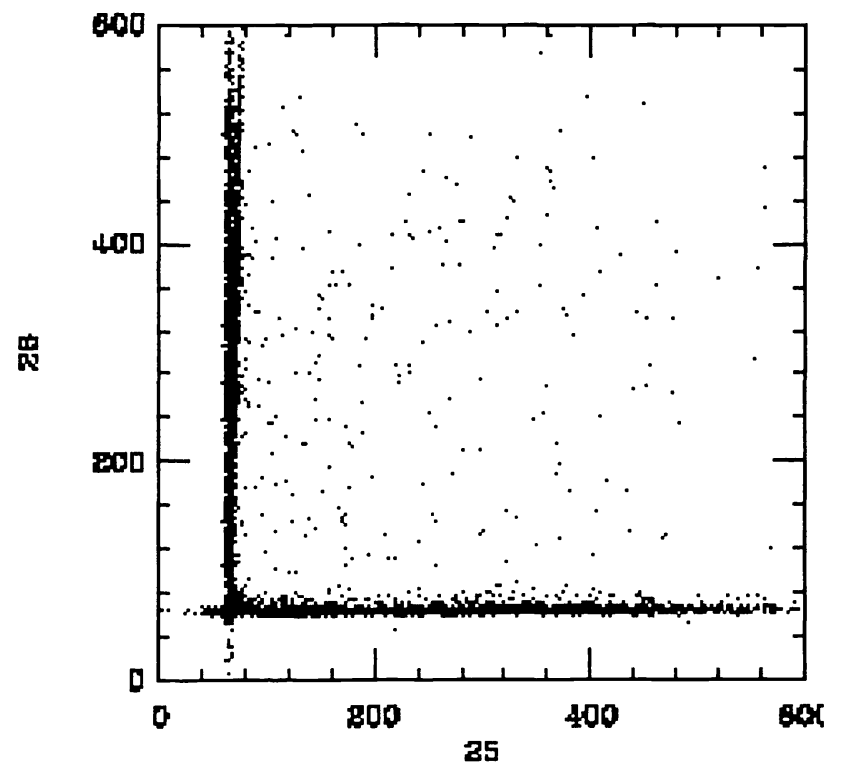

Figure 8 - Electrical cross-talk between two anodes

\subsubsection{Environmental Tests}

In order to consider the MAPMT for use in a space-borne instrument, it is necessary to ensure that it will survive the rigors of space flight. It was also subjected to two thermal-vacuum tests. The first test was performed on a bare tube in a vacuum of $10^{-7}$ Torr for two cycles between $-40^{\circ} \mathrm{C}$ and $+40^{\circ} \mathrm{C}$ with a day spent at each extreme for each cycle. The second test was performed on a tube potted in uralane with an operational base. The tube was kept in a vacuum of $10^{-7}$ Torr for two cycles between $-30^{\circ} \mathrm{C}$ and $+40^{\circ} \mathrm{C}$ with twelve hours at each extreme. The tube's gain was checked at several pressures ranging from $6.6 \times 10^{-7}$ Torr to $1.0 \times 10^{-4}$ Torr. No significant change in tube performance was observed for the range of temperature and vacuum covered by these tests.

The MAPMT was also subjected to vibration testing at the Marshall Space Flight Center in October 1998 to evaluate the structural design. The test conditions and procedures were consistent with NASA's General Environmental Vehicle Standards (GEVS). Two MAPMTs were tested: one bare tube and one with a bleeder string attached. Both tubes were potted in a test fixture. The potting was used to securely hold the MAPMT and to act as an electrical insulator around the 
metal exterior. All three principal axes were tested at two different vibration levels. The tubes were first tested to Delta II rocket vibration levels $(8.7 \mathrm{Grms})$ and then to general prototype qualifications levels (14.1 Grms). No variation in functional performance or vibrational characteristics occurred. Thus we conclude that the MAPMT, when properly mounted, is sufficiently rugged to survive launch loads. A vibration test of several MAPMTs with associated electronics and fiber connector is planned.

\section{SCINTILLATING FIBERS}

FiberGLAST utilizes the ability of scintillating fibers to detect ionizing particles in order to image the electromagnetic shower induced by gamma rays. Scintillating fibers have been under development at Washington University and UCLA/Fermilab for almost twenty years and have been used on numerous experiments. The CRIS instrument on the Advanced Composition Explorer (ACE) utilizes scintillating fibers to track cosmic rays and has operated successfully since its launch in August 1997 and is the first instrument to employ scintillating fibers in space (Stone 1998). Scintillating fibers have also been used in the high energy physics community and are currently being used in instruments including D0 and the CDF at Fermi National Laboratory. The FiberGLAST baseline instrument uses blue-emitting square scintillating fibers with a $0.75 \mathrm{~mm}$ cross section. There are several important issues in the use of scintillating fibers as detectors: light output, radiation damage effects and detection efficiency.

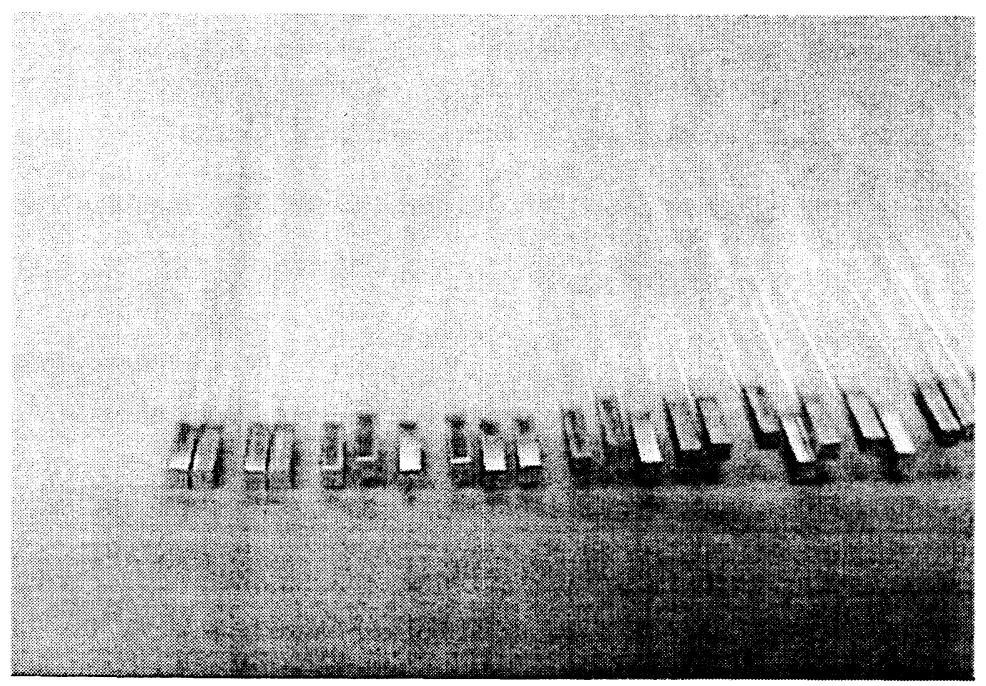

Figure 9 - Mirrored ends of square scintillating fibers

When an ionizing particle passes through a scintillating fiber the particle interacts with the polystyrene core doped with scintillating dyes and produces light. This light is internally reflected in the fiber and propagates along its length due to the index of refraction difference between the core and the claddings which surround the core. Multiclad fibers offer a significant improvement over singleclad fibers since a larger fraction of light is lightpiped. By applying a mirrored surface to the far end of a scintillating fiber, light can be reflected and transmitted back to the end with the photodetector. A variety of studies have been performed over the last year by the FiberGLAST team to improve the efficiency of these double-cladded, mirrored fibers. Working with the Bicron Corporation, we have developed fibers with sufficient light output for FiberGLAST (figure 9).

\subsection{Scintillating Fiber Radiation Damage}

Calculations using several computer codes predict that the scintillating fibers will receive a total dose of $\leq 1 \mathrm{krad}$ assuming a $550 \mathrm{~km}, 28.5$ degree inclination orbit during a ten year mission (Binns 1999). Most of this radiation will result from protons trapped in the South Atlantic Anomaly with energy of less than $30 \mathrm{MeV}$. This dose will be accumulated slowly over the course of the mission. Few studies have examined the effects of low level irradiation on scintillating fibers and none of these low radiation level studies have been done with blue-emitting fibers. FiberGLAST is currently baselining the use of blue emitting scintillating fibers of square cross section. Such fibers were used on the CRIS instrument on ACE which has 
received a dose of over $1 \mathrm{krad}$ of radiation since its 1997 launch in its orbit at L1. Most of this dose has been contributed by low energy protons $(\mathrm{E}<20 \mathrm{MeV})$ solar flares. No significant degradation in the performance and light output of the fibers has been observed. However, since there is presently no direct laboratory data on radiation damage of blue-emitting fibers from low level irradiation, we are currently carrying out a series of radiation damage tests on scintillating fibers using $68.8 \mathrm{MeV}$ protons at the Indiana University Cyclotron Facility. Results of these tests will be reported in a later paper.

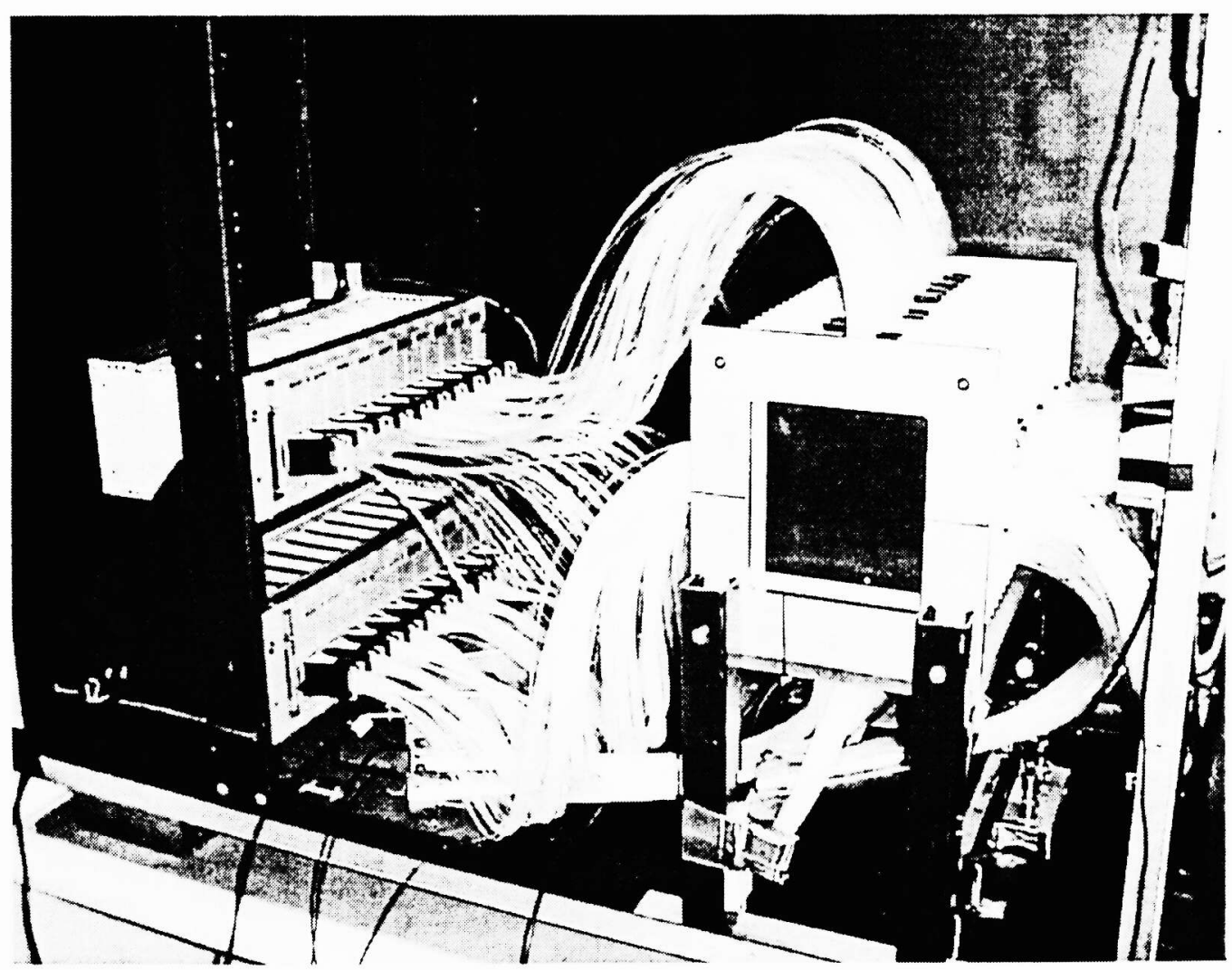

Figure 10 - Prototype Scintillating fiber/MAPMT system

\section{PRELIMINARY BEAM TEST RESULTS}

A prototype scintillating fiber/MAPMT system is presently being tested at the Center for Advanced Microstructures and Devices (CAMD) at Louisiana State University. A photon beam with a bremsstrahlung spectrum with an energy range between $50 \mathrm{MeV}$ and $1.5 \mathrm{GeV}$ is generated by the synchrotron electron ring. The system is composed of twenty $\mathrm{x}, \mathrm{y}$ planes (40) layers) of $0.75 \mathrm{~mm}$ scintillating fibers read out by 20 MAPMTs for a total of 1280 channels (figure 10). The spacing between these planes is $2 \mathrm{~cm}$. The 20 MAPMTs are read out using electronics which closely simulate the electronics being developed for the flight electronics. These ASIC simulator boards are composed of four boards, each of which reads sixteen MAPMT anodes. The anode signal is amplified and compared to a settable discriminator value. The hit pattern for each tube is then read out for each event. A 12-bit scalar is available for each channel as well as the OR of all sixteen channels on a board. The instrument is described in detail by Mallozzi et al. (1999). The system was operated in two modes: triggered externally by front and rear scintillators and self-triggered. The self-trigger mode can be set to several different logic configurations (e.g. 7 hit layers out of any 8 consecutive fiber layers). Figure 11 shows a self-triggered event's track in the Y-Z projection. 


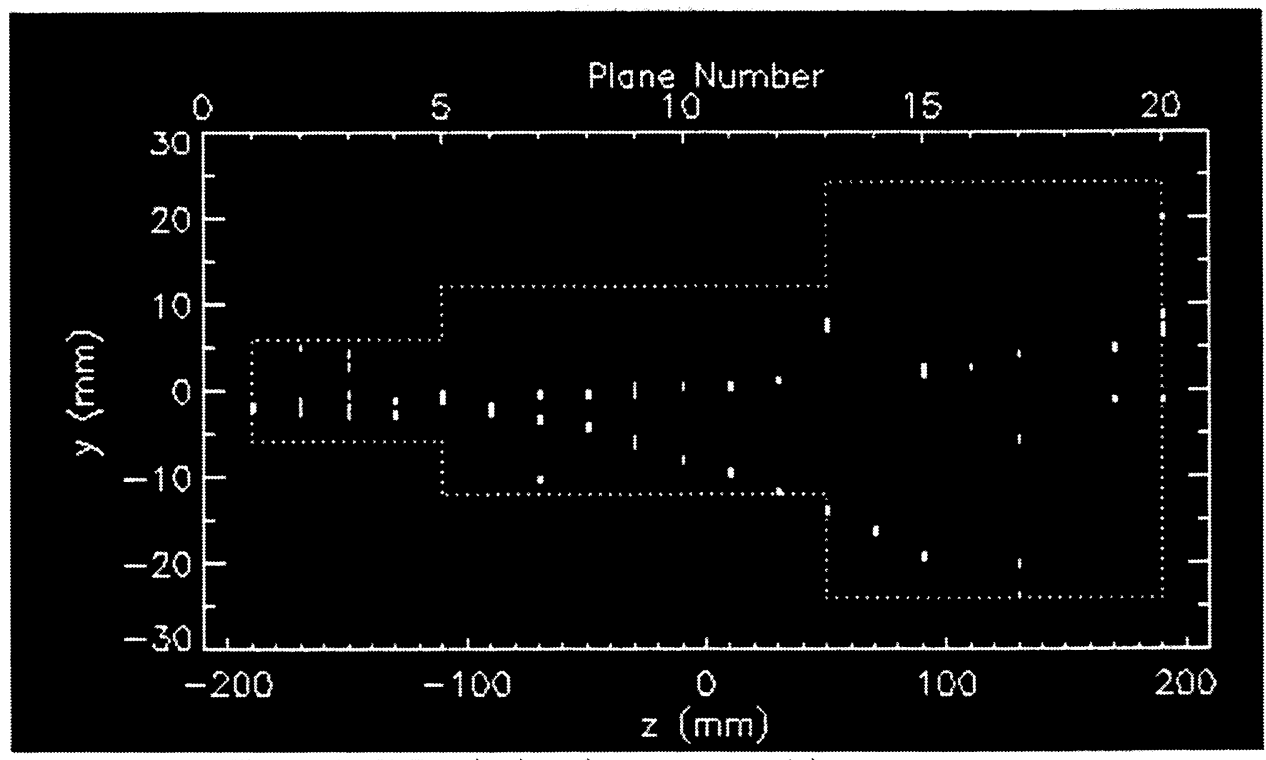

Figure $\| 1-Y-Z$ projection of gamma-ray track in prototype system

\section{PROTOTYPE ASIC DEVELOPMENT FOR MAPMT READOUT}

Because the front-end readout electronics for the MAPMTs in the FiberGLAST instrument must be physically located near the MAPMTs, for optimal performance and also owing to the large number of channels involved, very high density packaging is needed. The printed circuit board area available for the readout of each MAPMT is approximately $3.8 \mathrm{~cm} \mathrm{x}$ $4.3 \mathrm{~cm}$. In order to meet this requirement using the minimum amount of material and to stay within the front-end electronics power budget of $\sim 100 \mathrm{~W}$, an integrated circuit is needed. We are currently developing an ASIC that will integrate all the front-end electronics functions for 64 anodes (one MAPMT) at a power level of less than $250 \mu \mathrm{W}$ per channel for the tracker readout (an additional $50 \mu \mathrm{W}$ per channel is allocated for the pulse height analysis in the calorimeter section of the instrument).

Each MAPMT requires a discriminator with threshold set in the region of 0.3-0.5 photoelectrons. The thresholds must be set within $25 \%$ uniformity across all channels despite gain variations in the MAPMT. For that reason, the input stage of the ASIC features a 2-bit gain trim, adjustable on a per channel basis. The discriminator outputs identify the hit fibers in an event; sparse readout logic will present the channel numbers of the fibers over threshold to the data acquisition system. Additionally, a logical OR of all the discriminators in the ASIC is provided to the trigger system. The trigger decision will be based on coincidence and coarse-grained hit topology derived from the discriminator OR outputs from the ASIC's, together with information from the anti-coincidence system. It will also require additional information that will enable rejection of proton tracks that stop in the tracker. The latter mimic high-energy gamma events and cannot be distinguished based on coarse hit topology in the tracker and anti-coincidence system alone. Two approaches to the problem of rejecting this type of proton background are under consideration. The first is to add a second discriminator, with threshold set around 10-30 photoelectrons, to each channel, to distinguish the fibers in which a larger energy was deposited. The alternative approach is to add logic in the ASIC to derive additional trigger outputs which distinguish the case of an isolated hit or hits in a group of fibers from the case of a shower where many fibers relatively near to each other will be hit. The latter approach offers potential savings in power dissipation, but requires additional interconnect to carry the logic across ASIC boundaries.

The MAPMT readout ASIC will have internal data buffering and sparse readout logic, so that, after an event trigger has occurred, the data (channel numbers of the hit channels, and upper-level discriminator bits for these channels, if implemented) will be stored, freeing the input amplifier and discriminator resources to be reset, while the data is serially encoded and sent to the data acquisition system. Once the reset is complete, the detector can go live again, while the data readout is still in progress. 


\begin{tabular}{|l|l|}
\hline Power Dissipation (T/H powered on) (excluding Test Buffer) & $316 \mu \mathrm{W} / \mathrm{channel}$ \\
\hline Power Dissipation (T/H shutdown) & $231 \mu \mathrm{W} / \mathrm{channel}$ \\
\hline Input Amplifier Output Signal Range & $1.5 \mathrm{~V}$ \\
\hline Input Amplifier Feedback Capacitance & Selectable $4.52 \mathrm{pF}, 5.09 \mathrm{pF}, 5.59 \mathrm{pF}$, or $6.16 \mathrm{pF}$ \\
\hline Input Amplifier Risetime & $<100 \mathrm{~ns}$ \\
\hline Cross-talk / External Stray Capacitance between Channels & $<0.1 \% / \mathrm{pF}$ \\
\hline Discriminator Sensitivity & $<1 \mathrm{mV}$ \\
\hline Prop. Delay Input to Disc. Output @ 0.3 pe thr., 1 pe pulse & $200 \mathrm{~ns}$ \\
\hline Prop. Delay, Input to Disc. Output @ 0.3 pe thr., 0.4 pe pulse & $<600 \mathrm{~ns}$ \\
\hline Linearity (Input to Track/Hold Output) (0 to 1.5 V output) & $<2 \%$ \\
\hline Reset Pulse Width, Minimum & $<2 \mu \mathrm{s}$ \\
\hline Supply Voltage & $+3.0 \mathrm{~V}$ \\
\hline
\end{tabular}

Table 1. FiberGLAST Prototype ASIC Specifications

For the calorimeter section of the FiberGLAST instrument, pulse-height analysis will be used to provide additional energy information. The readout ASIC will have a track/hold circuit following the input amplifier, which, upon a trigger, will capture the pulse height out of the input amplifier, which will be a measure of the total charge from the corresponding anode. Afterwards, while the input amplifiers and discriminators are being reset for the next event, the sparse readout logic presents the analog values held by the track/hold circuits, of the hit channels only, to an A/D converter. The A/D converter may be integrated on the ASIC. The same ASIC will be used for both the tracker and calorimeter portions of the FiberGLAST instrument, so the pulse-height analysis circuitry will be configured so that it can be powered down in the tracker section, where it is not needed.

An 8-channel prototype ASIC is presently being fabricated. It has the input amplifier, track/hold circuit, and two discriminators on each channel; the trigger and sparse readout logic functions will be integrated into a companion FPGA for a complete functional prototype of the ASIC. Internal bias points of the prototype have been taken off-chip so that it can be run at various power levels and power/performance trade studies can be made. The prototype will be used in laboratory testing with the R5900-00-M64 MAPMT, and also is scheduled for use in a beam test of the instrument prototype in late 1999. Some key specifications of the prototype ASIC are given above in

Table 1.

\section{CONCLUSIONS}

The FiberGLAST collaboration has shown that scintillating fibers can be used successfully as a detector in the environment of space. Scintillating fibers have sufficient light output and detection efficiency. The multianode photomultiplier tube has been thoroughly tested and characterized. Its features include a low dark count which will provide a low occupancy rate for the instrument, sufficient gain to resolve single photoelectron events and allow for efficient selftriggering threshold of single photoelectron events, and low anode to anode cross-talk. Low powered electronics are now being fabricated which will meet all of the power, speed and triggering requirements for FiberGLAST. An instrument utilizing the scintillating fiber/MAPMT system has been tested and preliminary results show that the system performs well. It is clear that a scintillating fiber/MAPMT system is well matched for the demands of the GLAST mission: low power, low occupancy, and sufficient detection efficiency.

\section{ACKNOWLEDGMENTS}

The authors gratefully acknowledge support for this work through NASA grants NAG5-5112 and NAS 5-98036 and also wish to thank the operating staff at the CAMD facility for their help during the recent test of the prototype system.

\section{REFERENCES}

1. G.N. Pendleton, W.R. Binns, M.H, Israel, P.L. Hink, M.L. Cherry, W.S. Paciesas, R.M. Kippen, R.S. Mallozzi, T.A. Parnell, G.J. Fishman, T.O. Tumer, M.J. Christl, R.B. Wilson, J.H. Buckley, G. Richardson, S. Pengchamnan, K. Rielage, G. Karr, D. Wallace, J.M. Ryan, M.L. McConnell, and J.R. Macri, "FiberGLAST: a scintillating fiber approach to the GLAST mission," SPIE Proc., 3765, 1999. 
2. M. Enkelmann, U. Werthenbach, G. Zech, and T. Zeuner, "An optical readout for a fiber tracker," NIM A, 412, 216, 1998.

3. E. Stone, C.M.S. Cohen, W.R. Cook, A.C. Cummings, B. Gauld, B. Keckman, R.A. Leske, R.A. Mewaldt, M.R. Thayer, B.L. Dougherty, R.L. Grumm, B.D. Milliken, R.G. Radocinski, M.E. Wiedenbeck, E.R. Christian, S. Shuman, H. Trexel, T.T. Von Rosenvinge, W.R. Binns, D.J. Crary, P. Dowkontt, J. Epstein, P.L. Hink, J. Klarman, M. Lijowski, and M.A. Olevitch, "The Cosmic-ray Isotope Spectrometer for the Advanced Composition Explorer," Space Science Reviews, 86, $285,1998$.

4. W.R. Binns and the FiberGLAST Collaboration, "FiberGLAST Radiation Damage Report", Report to the GLAST Council, March 8, 1999.

5. R.S. Mallozzi, R.M. Kippen, G.N. Pendleton, W.S. Paciesas, G.A. Richardson, S. Pengchamnan, G. Karr, D. Wallace, G.J. Fishman, T.A. Parnell, R.B. Wilson, M.J. Christl, W.R. Binns, P.L. Hink, M.H. Israel, K.R. Rielage, J. Epstein, P. Dowkontt, J. Buckley, J. Ryan, J. Macri, M. McConnell, T. Tumer, M. Cherry, and M. Atac, "Beam test results for the FiberGLAST instrument," SPIE Proc., 3765, 1999. 